\section{Game, set and mismatch}

Peter Dearden

\author{
Mismatch: Why 0 ur World No Longer \\ Fits 0 ur Bodies \\ by Peter G luckman \& Mark Hanson \\ O xford U niversity Press, 0 xford, U K \\ 272 pp, \$30/£17 \\ ISBN 9780192806833
}

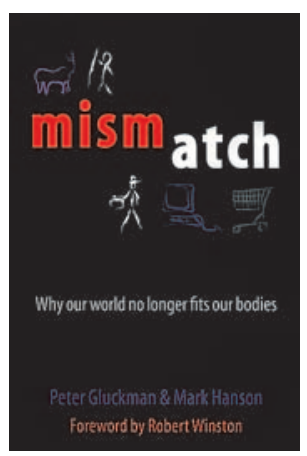

In recent years, studies of evolution and development have flourished, providing new insights into the mechanisms of evolution. O ne area that remains relatively unexplored, however, is the impact of evolution and development on human health. In Mismatch: Why O ur World No Longer Fits O ur Bodies, Peter Gluckman and Mark Hanson grapple with this idea to provide an 'evo-devo' model of many of the problems currently stalking the populations of the developed and developing worlds.

Gluckman and Hanson both have distinguished research careers focusing on 'predictive adaptive response'- the idea that the mammalian fetus uses information from the maternal environment to predict its postnatal environment and then changes its phenotype in response. The 'mismatch' of the book title refers to the idea that a mismatch between these predictions and the postnatal environment can lead to disease and other problems. The authors extend this idea and discuss more generally the idea of mismatch between humans and their environment as a cause of medical and societal problems.

The book is divided into two parts: match and mismatch. The first starts by providing lay descriptions of evolution and development to illustrate the concept of an organism being matched to its environment. In doing so, Mismatch provides an excellent description of predictive adaptive responses and explains some of the biology behind the concept. Gluckman and
Hanson imply that such mechanisms are just part of a continuum of developmental plasticity in all organisms that occurs in response to the environment. They point out that most studies of development are gene-centric and therefore try to ignore environmental influence. Although this is true, the gene-centric approach has provided powerful tools for dissecting development and these are now being used to examine environmental influences on development. The authors spend some time discussing heritable and long-term responses to the environment, and lead us through epigenetics and the phenomenon of DNA methylation. They describe well the control of gene expression through DNA methylation but miss, in some respects, other epigenetic effects. Despite this, $\mathrm{M}$ ismatch argues persuasively that the environmental inputs to development are important and somewhat overlooked

The authors then weave in the evolutionary story of H omo sapiens with emphasis on the environment in which our species has evolved, and how, over the last 10,000 years, that environment has been manipulated. The authors suggest that as the environment has been changed by humansthrough the advent of agriculture, urbanization, transport and so forth-that a mismatch has developed between our biology and our environment.

The second part of the book examines more closely the consequence of this mismatch in three areas important to human society and health. The first of these is the reduction in the age of female puberty that is occurring in the developed world. This is a problem for society because, as the authors explain, sexual maturity now precedes social maturity. Gluckman and Hanson suggest that this change might be related to improved maternal and postnatal nutrition, leading to a mismatch between biologythe ability to reproduce-and society.

The authors then present the case of 'metabolic disorder' - the increasing rates of diabetes and obesity in the developed and developing world-as a mismatch of fetal prediction with postnatal environment. The authors contend that this disorder is a normal part of human biology-a sort of human polyphenism that has evolved to cope with a nutrient-poor postnatal environment, which is now being triggered inappropriately by poor embryonic environments, or in individuals who developed in a constrained environment but then shifted to a rich postnatal environment.

Finally, the book examines ageing and menopause, again from the point of view of a mismatch. They argue that the diseases of ageing occur more frequently because our bodies are unable to cope comfortably with advanced age. This is due to increased longevity worldwide, which results from improved nutrition and a reduction in infectious disease.

The authors provide a short section discussing what might be done about these mismatches. M ost of what is suggested concerns early fetal life, such as ensuring correct and appropriate nutrition to pregnant mothers and improving the well-being of women in general. If, as Gluckman and Hanson persuasively argue, many modern ills spring from a mismatch between fetal predictions and postnatal life, then this seems to be a medically, morally and socially positive approach.

Gluckman and Hanson previously published a more technical description of their ideas in The Fetal M atrix (Cambridge University Press, Cambridge, UK), and $M$ ismatch covers similar ground. The difference is that Mismatch is written in an accessible style for a more general reader. The aim of this, I think, is a laudable one: to teach the general public about the idea of predictive adaptive response so that attitudes and behaviour around pregnancy might change. This more 'general science' pitch of $M$ ismatch means that many details are omitted, but the authors provide an extensive and usefully annotated bibliography.

M ismatch is a well-written book containing ideas that are important to our understanding of the relationship between human biology and environment. The range of examples and issues discussed-from goitre in Nepal to public health in ancient Rome, Lysenkoism to honey bees-provides an entertaining and stimulating read. Gluckman and $\mathrm{H}$ anson have presented ideas that might be crucial in tackling some of the problems of modern society.

\section{Peter Dearden ishead of the Laboratory for Evolution and Development at theUniversity of Otago in D unedin, New Zealand.}

E-mail: peter.dearden@stonebow.otago.ac.nz doi:10.1038/sj.embor.7400926 